\title{
Combination Treatment with Low-dose Niaspan and Tissue Plasminogen Activator Provides Neuroprotection after Embolic Stroke in Rats
}

\author{
Amjad Shehadah, MD ${ }^{1}$, Jieli Chen, MD $^{1,{ }^{*}}$, Yisheng Cui, MD ${ }^{1}$, Li Zhang, MD ${ }^{1}$, Cynthia \\ Roberts, BS ${ }^{1}$, Mei Lu, PhD ${ }^{2}$, and Michael Chopp, PhD $^{1,3}$ \\ ${ }^{1}$ Department of Neurology, Henry Ford Hospital, Detroit, MI, 48202 \\ 2Department of Biostatistics and Research Epidemiology, Henry Ford Hospital, Detroit, MI, 48202 \\ ${ }^{3}$ Department of Physics, Oakland University, Rochester, MI, 48309
}

\section{Abstract}

\begin{abstract}
Introduction-Niaspan, an extended-release formulation of Niacin (vitamin B3), has been widely used to increase high density lipoprotein (HDL) cholesterol and to prevent cardiovascular diseases and stroke. We have previously demonstrated that Niaspan $(40 \mathrm{mg} / \mathrm{kg})$ administered at 2 hours after stroke induces neuroprotection, while low dose Niaspan $(20 \mathrm{mg} / \mathrm{kg})$ does not reduce infarct volume. Tissue plasminogen activator (tPA) is an effective therapy for acute stroke, but its use remains limited by narrow therapeutic window. We have previously demonstrated that intravenous administration of tPA 4 hours after stroke in rats does not reduce infarct volume. In this study, we tested whether combination treatment with low-dose Niaspan $(20 \mathrm{mg} / \mathrm{kg})$ and tPA administered 4 hours after embolic stroke in a rat model reduces infarct volume and provides neuroprotection.
\end{abstract}

\begin{abstract}
Methods-Adult male Wistar rats were subjected to embolic middle cerebral artery occlusion (MCAo) and treated with low-dose Niaspan $(20 \mathrm{mg} / \mathrm{kg})$ alone $(\mathrm{n}=7)$, tPA $(10 \mathrm{mg} / \mathrm{kg})$ alone $(\mathrm{n}=7)$, combination of low-dose Niaspan and tPA $(n=7)$, or saline control $(n=9), 4$ hours after stroke. A battery of functional outcome tests was performed. Rats were sacrificed at 7 days after MCAo and lesion volumes were measured. To investigate the underlying mechanism of combination treatment neuroprotective effect, deoxynucleotidyl transferase-mediated dUTP nick-end labeling (TUNEL), cleaved caspase-3, tumor necrosis factor alpha (TNF-alpha), and toll-like receptor 4 (TLR-4) immunostaining were performed.
\end{abstract}

Results-Combination treatment with low-dose Niaspan and tPA significantly improved functional outcome compared to the saline control group ( $\mathrm{p}<0.05)$, while treatment with Niaspan or tPA alone did not significantly improve functional outcome compared to saline control group. Additionally, combination treatment significantly reduced infarct volume compared to saline control group $(\mathrm{p}=0.006)$ and infarct volume was significantly correlated with functional outcome

\footnotetext{
(C) 2011 Elsevier B.V. All rights reserved.

*Please send all correspondence to: Neurology Research Education \& Research Building, room 3091 Henry Ford Hospital 2799 West Grand Boulevard Detroit, MI 48202 Phone: (313) 916-1991 Fax: (313) 916-1318 jieli@neuro.hfh.edu.

Disclosures None.

Publisher's Disclaimer: This is a PDF file of an unedited manuscript that has been accepted for publication. As a service to our customers we are providing this early version of the manuscript. The manuscript will undergo copyediting, typesetting, and review of the resulting proof before it is published in its final citable form. Please note that during the production process errors may be discovered which could affect the content, and all legal disclaimers that apply to the journal pertain.
} 
$(\mathrm{p}=0.0008 ; \mathrm{r}=0.63)$. Monotherapy with Niaspan or tPA did not significantly decrease infarct volume compared to saline control group.

Combination treatment reduced apoptosis as measured by significant reduction in the number of TUNEL-positive cells and cleaved caspase- 3 expression in the ischemic brain compared to saline control group $(\mathrm{p}<0.05)$. Combination treatment also significantly reduced the expression of TNFalpha and TLR-4 in the ischemic brain compared to Niaspan, tPA and saline treatment groups $(\mathrm{p}<0.05)$. A significant interaction between Niaspan and tPA on the TNF-alpha expression was detected $(\mathrm{p}<0.05)$, indicating a synergy effect in the combination treatment group.

Conclusion-Treatment of stroke with combination of low-dose Niaspan and tPA at 4 hours after embolic stroke reduces infarct volume, improves neurological outcome and provides neuroprotection. The neuroprotective effects of combination treatment were associated with reduction of apoptosis and attenuation of TNF-alpha and TLR-4 expression.

\section{Keywords}

Niaspan; tPA; Neuroprotection; Apoptosis; TNF-alpha; TLR-4

\section{Introduction}

Niacin (nicotinic acid, vitamin B3) is the most potent available lipid-regulating agent to increase HDL levels [1]. Several clinical trials have demonstrated that niacin, alone or in combination with other lipid-lowering drugs, prevents cardiovascular diseases, stroke and atherosclerosis $[2,3]$. Niaspan, an extended-release formulation of Niacin, reduces the niacin-induced major side effects of flush and hepatotoxicity [4]. We have previously demonstrated that treatment of stroke with Niaspan $(40 \mathrm{mg} / \mathrm{kg})$ at 2 hours after middle cerebral artery occlusion (MCAo) reduces infarct volume, improves neurological outcome and provides neuroprotection [5], while low dose Niaspan $(20 \mathrm{mg} / \mathrm{kg})$ does not reduce infarct volume [5]. In order to test our hypothesis that combination treatment of experimental stroke with Niaspan and tPA reduces infarct volume and provides neuroprotection, we choose the low dose of Niaspan $(20 \mathrm{mg} / \mathrm{kg})$ since monotherapy with low dose Niaspan $(20 \mathrm{mg} / \mathrm{kg})$ does not reduce infarct volume.

Tissue plasminogen activator (tPA) is an effective therapy for acute stroke, but its use remains limited by narrow therapeutic window. We have previously demonstrated that intravenous administration of tPA 4 hours after stroke in rats does not reduce infarct volume $[6,7]$.

In this study, we tested whether combination treatment with low-dose Niaspan $(20 \mathrm{mg} / \mathrm{kg})$ and tPA administered 4 hours after embolic stroke in a rat model reduces infarct volume and provides neuroprotection.

Cerebral ischemia leads to neuronal damage through complex and interconnected pathophysiological events. Apoptosis and inflammation play key roles in the delayed injury in the penumbra $[8,9]$. Caspases, a family of cysteinyl-aspartate family, are essential players in apoptotic cell death after focal cerebral ischemia [10,11]. Administration of broad spectrum caspase inhibitors to ischemic rodents induced neuroprotection [12]. Caspase-3 is the most abundant caspase family member in the adult rodent brain [11]. Neurons are the predominant brain cells that undergo caspase-3-dependent apoptosis after hypoxia-ischemia [13].

There is increasing evidence that the inflammatory response in the surrounding of cerebral infarct contributes to the progression of the neuronal injury [14-16]. Tumor necrosis factor 
alpha (TNF-alpha) is one of the key immunomodulatory and pro-inflammatory cytokines up-regulated after focal stroke and plays a detrimental role in neuronal survival $[17,18]$. Inhibition of TNF-alpha effect in acute stroke have reduces the degree of ischemic injury in animal models [19], while administration of TNF-alpha during an ischemic brain insult has augments injury, as evidenced by increased tissue damage and neurological deficit [17].

Toll-like receptors (TLRs) are critical components of the innate immune system that mediate ischemic injury [20]. TLRs are present in the brain, where expression was believed to be limited to the glial cells [21]. However, recent evidence for the neuronal expression of TLRs has increased, suggesting a role during physiological as well as pathological condition [21, 22]. Several models of cerebral ischemia have suggested a role for TLR-4 in neuroinflammation and exacerbated stroke injury [23]. TLR-4 deficient mice subjected to MCAo exhibit improved neurological outcome and reduced infarct volumes, as well as reduced level of proinflammatory cytokines such as TNF-alpha and Interleukin-6 [24].

In the present study, we tested whether combination treatment with low-dose Niaspan $(20 \mathrm{mg} / \mathrm{kg})$ and $\mathrm{tPA}$ administered 4 hours after embolic stroke in a rat model provides neuroprotection as measured by neurological behavioral tests and infarct volume. We also investigated whether this neuroprotection is associated with reduction of apoptosis and attenuation of the inflammatory response in the ischemic brain.

\section{Materials and Methods}

\section{Animal middle cerebral artery occlusion (MCAO) model}

Male Wistar rats weighing 350 to $450 \mathrm{~g}$ were subjected to embolic middle cerebral artery occlusion (MCAo). [25]. Briefly, the animals were anesthetized with $4 \%$ isoflurane during induction and then maintained with $2 \%$ isoflurane in a mixture of $30 \% \mathrm{O} 2$ and $70 \% \mathrm{~N} 2 \mathrm{O}$. Body temperature was monitored and maintained at $37^{\circ} \mathrm{C}$ using a feedback-regulated water heating system. Under the operating microscope (Carl Zeiss), the right common carotid artery (CCA), the right external carotid artery (ECA), and the internal carotid artery (ICA) were isolated via a midline incision. A modified PE-50 catheter with a $0.3 \mathrm{~mm}$ outer diameter was gently advanced from the ECA into the lumen of the ICA until the tip of the catheter reach the origin of the MCA ( 15 to $16 \mathrm{~mm})$. Through the catheter, a single clot $(\sim 0.8 \mu \mathrm{L})$ along with 2 to $3 \mu \mathrm{L}$ of $0.9 \%$ saline was then gently injected. The catheter was withdrawn immediately after injection, and the right ECA was ligated.

\section{Experimental protocols}

Recombinant human t-PA (tPA, Genentech, San Francisco, CA, USA) was infused intravenously, through the tail vein, $4 \mathrm{~h}$ after ischemia at a dose of $10 \mathrm{mg} / \mathrm{kg}(10 \%$ bolus and the remainder at a continuous infusion over a 30-minutes interval using a syringe infusion pump; Harvard Apparatus, Holliston, MA, USA). This dose of tPA $(10 \mathrm{mg} / \mathrm{kg})$ is commonly used for investigating the effect of fibrinolysis in rodents [26].

Niaspan (dissolved in water; Kos Pharmaceuticals, Canburry, NJ) was gavaged at dose of 20 $\mathrm{mg} / \mathrm{kg}, 4$ hours after embolic MCAo and was followed by a second dose 24 hours after MCAo and daily for 7 days.

Four hours after the MCAo, rats were randomly separated into 4 groups:

1. Saline control: animals were infused with saline at 4 hours after MCAo $(n=9)$.

2. Monotherapy with intravenous tPA $(10 \mathrm{mg} / \mathrm{kg})$ at 4 hours after MCAo $(\mathrm{n}=7)$. 
3. Monotherapy with oral Niaspan $20 \mathrm{mg} / \mathrm{kg}$ at 4 hours after MCAo and daily for 7 days $(n=7)$.

4. Combination treatment with $\mathrm{tPA}(10 \mathrm{mg} / \mathrm{kg})$ once at 4 hours after MCAo and oral Niaspan $20 \mathrm{mg} / \mathrm{kg}$ at 4 hours after MCAo and daily for 7 days $(\mathrm{n}=7)$.

All rats were sacrificed at 7 days after MCAo.

\section{Functional tests}

A modified neurological severity score (mNSS) and Foot-fault test were performed before MCAo, and at 1, 3 and 7 days after MCAo by an investigator who was blinded to the experimental groups.

Modified neurological severity score (mNSS)-Neurological function was graded on a scale of 0 to 18 (normal score, 0 ; maximal deficit score, 18). mNSS is a composite of motor, sensory, reflex, and balance tests. In the severity scores of injury, 1 score point was awarded for the inability to perform the test or for the lack of a tested reflex; thus, the higher score, the more severe is the injury [27].

Foot-fault test-Foot-fault test is tested for placement dysfunction of forelimbs. Animals were placed on an elevated grid floor $(45 \mathrm{~cm} \times 30 \mathrm{~cm}), 2.5 \mathrm{~cm}$ higher than a solid base floor, with $2.5 \mathrm{~cm} \times 2.5 \mathrm{~cm}$ diameter openings. Animals tend to move on the grid with their paws placed on the wire frame. When animals inaccurately place a paw, the front limb falls through one of the openings in the grid. When the paw fall or slip between the wire, this was recorded as a foot fault. Total 100 of steps (movement of each forelimb) were counted, and the total number of foot faults for left forelimb was recorded. The percentage of foot fault of left paw to total steps are calculated [28, 29].

\section{Histological and immunohistochemical assessment}

At seven days after MCAo, animals were sacrificed and brains were fixed by transcardial perfusion with saline, followed by perfusion and immersion in $4 \%$ paraformaldehyde before being embedded in paraffin. Seven coronal sections of tissue were processed and stained with hematoxylin and eosin (H\&E) for calculation of volume of cerebral infarction [30]. The indirect lesion area, in which the intact area of the ipsilateral hemisphere was subtracted from the area of the contralateral hemisphere, was calculated using the Global Lab Image analysis system (Data Translation, Malboro, MA) [30]. Lesion volume is presented as a volume percentage of the lesion compared with the contralateral hemisphere. Gross hemorrhage, defined as blood evident to the unaided eye on the H\&E stained coronal sections, was evaluated on seven H\&E stained coronal sections for each animal [31]. The incidence of gross hemorrhage is presented as the percentage of animals per group with identified gross hemorrhage on any coronal section.

\section{Immunohistochemical staining}

A standard paraffin block was obtained from the center of the lesion (bregma $-1 \mathrm{~mm}$ to +1 $\mathrm{mm})$. A series of $6 \mu \mathrm{m}$ thick sections were cut from the block. Every $10^{\text {th }}$ coronal section for a total 5 sections was used for immunohistochemical staining. Antibody against cleaved caspase-3 (rabbit polyclonal IgG; dilution 1:200, Cell Signaling Technology, Danvers, Massachusetts), TNF-alpha (rabbit polyclonal IgG; dilution 1:200; Abcam PLC, Cambridge, Massachusetts ), and TLR-4 ( goat polyclonal IgG; dilution 1:100; Cruz Biotech Inc, Santa Cruz, California) immunostaining were performed. Control experiments consisted of staining brain coronal tissue sections as outlined above, but the primary antibodies were omitted, as previously described [32]. 
Terminal deoxynucleotidyl transferase-mediated dUTP nick-end labeling (TUNEL) for measuring apoptosis was performed using a commercial kit (ApopTag kit, Chemicon, S7100).

\section{Quantitation}

For measurement of apoptotic markers, each cleaved caspase-3 and TUNEL immunostained coronal section was digitized using a 20X objective, via the MCID computer imaging analysis system. In order to minimize the potential effect of infarct volume reduction on the tested parameters, five sections from the standard reference coronal section and 8 brain fields within each section were acquired and the total number of cleaved caspase- 3 and TUNEL positive cells in the 8 fields of the ischemic border area (IBZ),adjacent to the ischemic core, were counted using the MCID computer imaging analysis system (Imaging Research, St. Catharines, Canada). The total number of positive cells per $\mathrm{mm}^{2}$ area is presented.

For quantitative measurements of TNF-alpha and TLR-4, five slides from each brain, with each slide containing 8 fields from the IBZ were digitized under a 40x objective (Olympus BX40) using a 3-CCD color video camera (Sony DXC-970MD) interfaced with an MCID image analysis system (Imaging Research, St. Catharines, Canada) [33-35]. Data were analyzed in a blinded manner and presented as percentage of positive area for, TNF-alpha and TLR-4, respectively.

\section{Statistical Analysis}

Animals subjected to MCAo were randomized into one of four groups including saline control group, Monotherapy with tPA $10 \mathrm{mg} / \mathrm{kg}$, Monotherapy with Niaspan $20 \mathrm{mg} / \mathrm{kg}$, and Combination treatment with Niaspan $20 \mathrm{mg} / \mathrm{kg}$ and tPA $10 \mathrm{mg} / \mathrm{kg}$. Behavior tests (mNSS and foot-fault test) were performed before MCAo at 1, 3 and 7 days after MCAo. Considering a two-sided test and $\alpha=0.05$, the sample size of the experimental groups was planned to have $80 \%$ power to detect the difference among the groups with an effect size

$d=1.46, \quad\left(d=\frac{M_{\max }-M_{\min }}{\sigma}\right)$, assuming equal space of means.

The study was designed to test the combination treatment effect on the functional recovery, measured from two behavioral tests at day 7 as the primary outcome, at day 3 and the treatment effect on histological measurements as secondary outcomes. Data were evaluated for normality. Data transformation was considered if data were not normal. Since data were not normal, ranked data were used for the analysis.

The global test using Generalized Estimating Equations (GEE) [36] was used to test the group difference on functional recovery measured from multiple behavior tests. The analysis was started by testing the treatment (Niaspan and tPA) interaction, followed by testing the subgroup analysis, if the interaction or main effect was detected at the 0.05 level. The Global Test on multiple outcomes was considered more efficient than a single outcome, when the group effects were consistent on all the outcomes (e.g. positive correlation). The significant treatment interaction may indicate synergy effects of the two treatments and no interaction indicates the additive effects of the two treatments. Two-way ANOVA was used to test the effect of combination treatment on infarct volume and Immunostaining measures including cleaved caspase-3, TUNEL, TNF-alpha, and TLR-4 using similar approach.

Spearman correlation coefficients were calculated to study the correlation between functional tests at day 7 and the histological and the immunohistochemical measurements. 


\section{Results}

\section{Combination treatment with Niaspan and tPA, improves neurological outcome and reduces infarct volume without increasing the incidence of gross hemorrhage}

The mortality rates after stroke were $11 \%$ (one of 9) in saline control group, $14 \%$ (one of 7) in Niaspan monotherapy group, $14 \%$ (one of 7) in tPA monotherapy group and $0 \%$ in the combination treatment group. Physiological parameters of all analyzed animals were comparable prior and after MCAo.

To test the effect of the combination treatment of stroke rats on functional outcome, GEE statistical analysis was performed to detect the differences in functional recovery among the 4 groups (saline control, Niaspan monotherapy, tPA monotherapy and combination treatment). On day 7, No treatment interaction was detected based on the global test $(\mathrm{p}=0.81)$ indicating additivity of the two treatments (Niaspan, tPA). The global test showed that combination treatment with Niaspan and tPA significantly improved functional outcome at day 7 compared to saline control group $(\mathrm{p}<0.05)$, while treatment with Niaspan or tPA alone did not significantly improve functional outcome compared to saline control group. Additionally, combination treatment significantly improved functional recovery compared with saline control in a modified Neurological Severity Score ( $\mathrm{p}<0.05$; Figure $1 \mathrm{~A})$ and footfault test $(\mathrm{p}<0.05$; Figure 1B).

On day 3, combination treatment significantly improved functional recovery compared with saline control in a foot-fault test ( $<<0.05$; Figure 1B) and marginally improved mNSS compared with saline control group $(\mathrm{p}=0.06)$.

The combination treatment did not increase the incidence of gross hemorrhage $(14 \%, 1$ out of 7) compared to that in Niaspan monotherapy (14\%, 1 out of 7), tPA monotherapy (14\%, 1 out of 7$)$ and saline control $(11 \%, 1$ out of 9$)$.

\section{Lesion Volume (Figure 1C)}

Two-way ANOVA was used to test the differences in ischemic lesion volume between treatment groups. Combination treatment significantly reduced infarct volume compared to saline control group ( $\mathrm{p}=0.0006)$ or tPA monotherapy $(\mathrm{p}=0.006)$, and marginally compared to Niaspan monotherapy $(\mathrm{p}=0.08)$. Additionally, infarct volume was significantly correlated with functional outcome ( $\mathrm{p}=0.0008 ; \mathrm{r}=0.63$ ). Monotherapy with Niaspan or tPA did not significantly decrease infarct volume compared to saline control group.

\section{Combination treatment decreases the expression of TUNEL and cleaved caspase-3}

To test whether combination treatment regulates apoptosis, two apoptotic markers, TUNEL and cleaved caspase-3, were employed. Figure 2A-B show that combination treatment significantly decreased the number of immunoreactive TUNEL-positive cells in the ischemic brain compared to saline treated rats $(\mathrm{p}<0.05)$, as well as combination treatment significantly decreased the number of cleaved caspase-3 positive cells compared with saline control rats $(\mathrm{p}<0.0001)$ or with the tPA monotherapy group $(\mathrm{p}<0.05)$. We also observed that Niaspan treatment group significantly reduced the number of cleaved caspase-3 positive cells compared to saline control $(\mathrm{p}=0.0003)$. These data indicate that combination treatment has an antiapoptotic role in the treatment of stroke.

\section{Combination treatment attenuates the expression of TNF-alpha, and TLR-4 in the ischemic brain}

In order investigate the effect of combination treatment on the inflammatory reaction in the ischemic brain, we measured the expression of TNF-alpha in the ipsilateral ischemic border 
area. Figure $2 \mathrm{C}$ shows that TNF-alpha expression decreased in the combination treatment group compared with tPA $(\mathrm{p}<0.0001)$ or Niaspan monotherapy $(\mathrm{p}=0.006)$, as well as with saline control $(\mathrm{p}<0.0001)$. A significant interaction between Niaspan and TPA on the TNFalpha expression was detected $(\mathrm{p}<0.05)$, indicating a synergy effect in the combination treatment group.

To further investigate mechanisms underlying the Niaspan-induced neuroprotection after stroke, TLR-4 expression was measured in the ischemic border. Figure 2D shows that combination treatment significantly decreased TLR- 4 expression in the ischemic brain compared with tPA $(\mathrm{p}=0.024)$ or Niaspan $(\mathrm{p}=0.0015)$ monotherapy as well as with saline control $(\mathrm{p}=0.0003)$.

\section{Discussion}

The results of the present study indicate that treatment of stroke with combination of lowdose Niaspan and tPA at 4 hours after embolic stroke in the rat reduces infarct volume, improves neurological outcome and provides neuroprotection. Combination treatment significantly reduced apoptosis as evidenced by reduction of TUNEL and caspase-3 immunoreactive positive cells in the ischemic brain. Concomitant with the neuroprotective effects in the combination treatment, is a significant attenuation of the TNF-alpha and TLR-4 expression in the ischemic brain.

\section{Combination treatment decreases the expression of TUNEL and cleaved}

Apoptosis contributes to ischemic cell damage after stroke [37]. Caspase-3, an executioner caspase, is present in a wide variety of cells [13]. During ischemia, caspase-3 is cleaved and activated where upon it degrades multiple substrates in the cytoplasm and nucleus leading to cell death [38]. Caspase-3 deficient adult mice were more resistant to ischemic stress both in vivo and in vitro.[38]. Treatment with caspase-3 inhibitors reduced ischemic-induced brain damage [39, 40]. Moreover, inhibition of Caspase-3 like protease activity prevented DNA fragmentation in the ischemic brain [41].

Our data demonstrate that combination treatment inhibits cleaved caspase-3 expression and decreases DNA fragmentation in the brain after embolic stroke. Therefore, combination treatment has an anti-apoptotic role in the treatment of stroke in rats.

\section{Combination treatment attenuates the expression of TNF-alpha, and TLR-4 in the ischemic brain}

The inflammatory, innate and adaptive immune mechanisms participate in many facets of vascular disease [15]. The brain inflammatory response to injury is mediated in part by the proinflammatory cytokines such as TNF-alpha [14]. Administration of recombinant TNFalpha excacerbated ischemic brain injury as evidenced by increased tissue damage and neurological deficits [42]. Correspondingly, inhibition of TNF-alpha using an anti-TNF neutralizing antibody [43] or inhibition of soluble TNF-alpha receptor type1 [44] reduced ischemic damage and improved functional outcome after stroke [17].

It is increasingly clear that post-stroke neuroinflammation from TLR-4 signaling worsens stroke outcome, as measured by infarct volumes, neurological function and inflammatory markers [23]. Activation of TLR-4 signaling contributes to hippocampal neuronal death following global cerebral ischemia/reperfusion [45], while TLR-4 deficient mice have lower infarct volumes, better outcomes in neurological and behavioral tests, and less inflammatory response after an ischemic insult [46]. Additionally, TLR-4 deficiency protects mice against focal ischemia and axotomy-induced retinal ganglion cell degeneration [47]. 
Our data suggest that combination treatment with Niaspan and tPA reduces the inflammatory response after embolic stroke in rats as was demonstrated by reduction of TNF-alpha and TLR-4 expression in the ischemic brain.

Effects of neuroprotectant drugs may be influenced by the occurrence of reperfusion; however, cerebral blood flow monitoring was not performed which could be a limitation of this study.

In summary, we demonstrated that treatment of stroke with combination of low-dose Niaspan and tPA at 4 hours after embolic stroke reduces infarct volume, improves neurological outcome and provides neuroprotection. The neuroprotective effects of combination treatment were associated with reduction of apoptosis and attenuation of TNFalpha and TLR-4 expression in the ischemic brain.

\section{Acknowledgments}

The authors wish to thank Genentech, Inc. for providing recombinant human t-PA. We also thank Qinge Lu and Supata Santra for technical assistance.

Sources of Funding This work was supported by National Institute on Aging RO1 AG031811 (JC), National Institute of Neurological Disorders and Stroke PO1 NS23393 (MC) and 1R41NS064708 (JC), and American Heart Association grant 09GRNT2300151 (JC).

\section{References}

1. Chapman MJ, Assmann G, Fruchart JC, Shepherd J, Sirtori C. Raising high-density lipoprotein cholesterol with reduction of cardiovascular risk: the role of nicotinic acid--a position paper developed by the European Consensus Panel on HDL-C. Curr Med Res Opin. 2004; 20(8):12531268. [PubMed: 15324528]

2. Meyers CD, Kamanna VS, Kashyap ML. Niacin therapy in atherosclerosis. Curr Opin Lipidol. 2004; 15(6):659-665. [PubMed: 15529025]

3. Singh IM, Shishehbor MH, Ansell BJ. High-density lipoprotein as a therapeutic target: a systematic review. JAMA. 2007; 298(7):786-798. [PubMed: 17699012]

4. Carlson LA. Niaspan, the prolonged release preparation of nicotinic acid (niacin), the broadspectrum lipid drug. Int J Clin Pract. 2004; 58(7):706-713. [PubMed: 15311728]

5. Shehadah A, Chen J, Zacharek A, Cui Y, Ion M, Roberts C, Kapke A, Chopp M. Niaspan treatment induces neuroprotection after stroke. Neurobiol Dis. 40(1):277-283. [PubMed: 20554037]

6. Zhang Z, Zhang L, Yepes M, Jiang Q, Li Q, Arniego P, Coleman TA, Lawrence DA, Chopp M. Adjuvant treatment with neuroserpin increases the therapeutic window for tissue-type plasminogen activator administration in a rat model of embolic stroke. Circulation. 2002; 106(6):740-745. [PubMed: 12163437]

7. Zhang L, Zhang ZG, Ding GL, Jiang Q, Liu X, Meng H, Hozeska A, Zhang C, Li L, Morris D, et al. Multitargeted effects of statin-enhanced thrombolytic therapy for stroke with recombinant human tissue-type plasminogen activator in the rat. Circulation. 2005; 112(22):3486-3494. [PubMed: 16316970]

8. Graham SH, Chen J. Programmed cell death in cerebral ischemia. J Cereb Blood Flow Metab. 2001; 21(2):99-109. [PubMed: 11176275]

9. Becker KJ. Inflammation and acute stroke. Curr Opin Neurol. 1998; 11(1):45-49. [PubMed: 9484616]

10. Schulz JB, Weller M, Moskowitz MA. Caspases as treatment targets in stroke and neurodegenerative diseases. Ann Neurol. 1999; 45(4):421-429. [PubMed: 10211465]

11. Namura S, Zhu J, Fink K, Endres M, Srinivasan A, Tomaselli KJ, Yuan J, Moskowitz MA. Activation and cleavage of caspase- 3 in apoptosis induced by experimental cerebral ischemia. $\mathrm{J}$ Neurosci. 1998; 18(10):3659-3668. [PubMed: 9570797] 
12. Rabuffetti M, Sciorati C, Tarozzo G, Clementi E, Manfredi AA, Beltramo M. Inhibition of caspase-1-like activity by Ac-Tyr-Val-Ala-Asp-chloromethyl ketone induces long-lasting neuroprotection in cerebral ischemia through apoptosis reduction and decrease of proinflammatory cytokines. J Neurosci. 2000; 20(12):4398-4404. [PubMed: 10844008]

13. Manabat C, Han BH, Wendland M, Derugin N, Fox CK, Choi J, Holtzman DM, Ferriero DM, Vexler ZS. Reperfusion differentially induces caspase-3 activation in ischemic core and penumbra after stroke in immature brain. Stroke. 2003; 34(1):207-213. [PubMed: 12511776]

14. Barone FC, Feuerstein GZ. Inflammatory mediators and stroke: new opportunities for novel therapeutics. J Cereb Blood Flow Metab. 1999; 19(8):819-834. [PubMed: 10458589]

15. Chamorro A, Hallenbeck J. The harms and benefits of inflammatory and immune responses in vascular disease. Stroke. 2006; 37(2):291-293. [PubMed: 16410468]

16. Wang Q, Tang XN, Yenari MA. The inflammatory response in stroke. J Neuroimmunol. 2007; 184(1-2):53-68. [PubMed: 17188755]

17. Wang X, Feuerstein GZ, Xu L, Wang H, Schumacher WA, Ogletree ML, Taub R, Duan JJ, Decicco CP, Liu RQ. Inhibition of tumor necrosis factor-alpha-converting enzyme by a selective antagonist protects brain from focal ischemic injury in rats. Mol Pharmacol. 2004; 65(4):890-896. [PubMed: 15044618]

18. Hallenbeck JM. The many faces of tumor necrosis factor in stroke. Nat Med. 2002; 8(12):13631368. [PubMed: 12457181]

19. Shohami E, Ginis I, Hallenbeck JM. Dual role of tumor necrosis factor alpha in brain injury. Cytokine Growth Factor Rev. 1999; 10(2):119-130. [PubMed: 10743503]

20. Marsh BJ, Williams-Karnesky RL, Stenzel-Poore MP. Toll-like receptor signaling in endogenous neuroprotection and stroke. Neuroscience. 2009; 158(3):1007-1020. [PubMed: 18809468]

21. Katsargyris A, Klonaris C, Alexandrou A, Giakoustidis AE, Vasileiou I, Theocharis S. Toll-like receptors in liver ischemia reperfusion injury: a novel target for therapeutic modulation? Expert Opin Ther Targets. 2009; 13(4):427-442. [PubMed: 19335065]

22. Tang SC, Arumugam TV, Xu X, Cheng A, Mughal MR, Jo DG, Lathia JD, Siler DA, Chigurupati S, Ouyang X, et al. Pivotal role for neuronal Toll-like receptors in ischemic brain injury and functional deficits. Proc Natl Acad Sci U S A. 2007; 104(34):13798-13803. [PubMed: 17693552]

23. Buchanan MM, Hutchinson M, Watkins LR, Yin H. Toll-like receptor 4 in CNS pathologies. J Neurochem. 114(1):13-27. [PubMed: 20402965]

24. Cao CX, Yang QW, Lv FL, Cui J, Fu HB, Wang JZ. Reduced cerebral ischemia-reperfusion injury in Toll-like receptor 4 deficient mice. Biochem Biophys Res Commun. 2007; 353(2):509-514. [PubMed: 17188246]

25. Zhang RL, Chopp M, Zhang ZG, Jiang Q, Ewing JR. A rat model of focal embolic cerebral ischemia. Brain Res. 1997; 766(1-2):83-92. [PubMed: 9359590]

26. Niessen F, Hilger T, Hoehn M, Hossmann KA. Thrombolytic treatment of clot embolism in rat: comparison of intra-arterial and intravenous application of recombinant tissue plasminogen activator. Stroke. 2002; 33(12):2999-3005. [PubMed: 12468803]

27. Chen J, Li Y, Wang L, Lu M, Zhang X, Chopp M. Therapeutic benefit of intracerebral transplantation of bone marrow stromal cells after cerebral ischemia in rats. J Neurol Sci. 2001; 189(1-2):49-57. [PubMed: 11535233]

28. Barth TM, Grant ML, Schallert T. Effects of MK-801 on recovery from sensorimotor cortex lesions. Stroke. 1990; 21(11 Suppl):III153-157. [PubMed: 2237974]

29. Schallert T, Fleming SM, Leasure JL, Tillerson JL, Bland ST. CNS plasticity and assessment of forelimb sensorimotor outcome in unilateral rat models of stroke, cortical ablation, parkinsonism and spinal cord injury. Neuropharmacology. 2000; 39(5):777-787. [PubMed: 10699444]

30. Swanson RA, Morton MT, Tsao-Wu G, Savalos RA, Davidson C, Sharp FR. A semiautomated method for measuring brain infarct volume. J Cereb Blood Flow Metab. 1990; 10(2):290-293. [PubMed: 1689322]

31. Zhang L, Chopp M, Jia L, Cui Y, Lu M, Zhang ZG. Atorvastatin extends the therapeutic window for tPA to $6 \mathrm{~h}$ after the onset of embolic stroke in rats. J Cereb Blood Flow Metab. 2009; 29(11): 1816-1824. [PubMed: 19638998] 
32. Li Y, Jiang N, Powers C, Chopp M. Neuronal damage and plasticity identified by microtubuleassociated protein 2, growth-associated protein 43, and cyclin D1 immunoreactivity after focal cerebral ischemia in rats. Stroke. 1998; 29(9):1972-1980. discussion 1980-1971. [PubMed: 9731626]

33. Chen J, Zhang ZG, Li Y, Wang L, Xu YX, Gautam SC, Lu M, Zhu Z, Chopp M. Intravenous administration of human bone marrow stromal cells induces angiogenesis in the ischemic boundary zone after stroke in rats. Circ Res. 2003; 92(6):692-699. [PubMed: 12609969]

34. Calza L, Giardino L, Giuliani A, Aloe L, Levi-Montalcini R. Nerve growth factor control of neuronal expression of angiogenetic and vasoactive factors. Proc Natl Acad Sci U S A. 2001; 98(7):4160-4165. [PubMed: 11259645]

35. Chen J, Zhang ZG, Li Y, Wang Y, Wang L, Jiang H, Zhang C, Lu M, Katakowski M, Feldkamp $\mathrm{CS}$, et al. Statins induce angiogenesis, neurogenesis, and synaptogenesis after stroke. Ann Neurol. 2003; 53(6):743-751. [PubMed: 12783420]

36. Lu M, Chen J, Lu D, Yi L, Mahmood A, Chopp M. Global test statistics for treatment effect of stroke and traumatic brain injury in rats with administration of bone marrow stromal cells. $\mathrm{J}$ Neurosci Methods. 2003; 128(1-2):183-190. [PubMed: 12948561]

37. Savitz SI, Erhardt JA, Anthony JV, Gupta G, Li X, Barone FC, Rosenbaum DM. The novel betablocker, carvedilol, provides neuroprotection in transient focal stroke. J Cereb Blood Flow Metab. 2000; 20(8):1197-1204. [PubMed: 10950380]

38. Le DA, Wu Y, Huang Z, Matsushita K, Plesnila N, Augustinack JC, Hyman BT, Yuan J, Kuida K, Flavell RA, et al. Caspase activation and neuroprotection in caspase-3- deficient mice after in vivo cerebral ischemia and in vitro oxygen glucose deprivation. Proc Natl Acad Sci U S A. 2002; 99(23):15188-15193. [PubMed: 12415117]

39. Sun M, Xu C. Neuroprotective mechanism of taurine due to up-regulating calpastatin and downregulating calpain and caspase-3 during focal cerebral ischemia. Cell Mol Neurobiol. 2008; 28(4): 593-611. [PubMed: 17712625]

40. Xu J, Culman J, Blume A, Brecht S, Gohlke P. Chronic treatment with a low dose of lithium protects the brain against ischemic injury by reducing apoptotic death. Stroke. 2003; 34(5):12871292. [PubMed: 12677021]

41. Luo Y, Cao G, Pei W, O’Horo C, Graham SH, Chen J. Induction of caspase-activated deoxyribonuclease activity after focal cerebral ischemia and reperfusion. J Cereb Blood Flow Metab. 2002; 22(1):15-20. [PubMed: 11807389]

42. Barone FC, Arvin B, White RF, Miller A, Webb CL, Willette RN, Lysko PG, Feuerstein GZ. Tumor necrosis factor-alpha. A mediator of focal ischemic brain injury. Stroke. 1997; 28(6):12331244. [PubMed: 9183357]

43. Hosomi N, Ban CR, Naya T, Takahashi T, Guo P, Song XY, Kohno M. Tumor necrosis factoralpha neutralization reduced cerebral edema through inhibition of matrix metalloproteinase production after transient focal cerebral ischemia. J Cereb Blood Flow Metab. 2005; 25(8):959_ 967. [PubMed: 15729288]

44. Nawashiro H, Martin D, Hallenbeck JM. Inhibition of tumor necrosis factor and amelioration of brain infarction in mice. J Cereb Blood Flow Metab. 1997; 17(2):229-232. [PubMed: 9040503]

45. Hua F, Ma J, Ha T, Xia Y, Kelley J, Williams DL, Kao RL, Browder IW, Schweitzer JB, Kalbfleisch JH, et al. Activation of Toll-like receptor 4 signaling contributes to hippocampal neuronal death following global cerebral ischemia/reperfusion. J Neuroimmunol. 2007; 190(1-2): 101-111. [PubMed: 17884182]

46. Caso JR, Pradillo JM, Hurtado O, Lorenzo P, Moro MA, Lizasoain I. Toll-like receptor 4 is involved in brain damage and inflammation after experimental stroke. Circulation. 2007; 115(12): 1599-1608. [PubMed: 17372179]

47. Kilic U, Kilic E, Matter CM, Bassetti CL, Hermann DM. TLR-4 deficiency protects against focal cerebral ischemia and axotomy-induced neurodegeneration. Neurobiol Dis. 2008; 31(1):33-40. [PubMed: 18486483] 

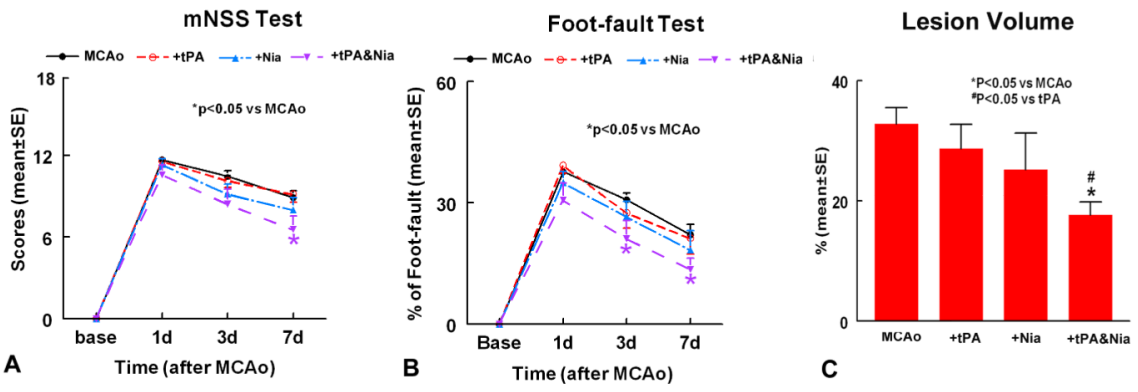

Figure 1. Neurological outcome and lesion volume measurements after stroke Panels A-B show mNSS (A) and foot-fault (B) tests after stroke in the 4 experimental groups (MCAo control, tPA-10mg $/ \mathrm{kg}$, Niaspan-20mg $/ \mathrm{kg}$, and Combination treatment tPA and Niaspan treatment groups). Panel $\mathbf{C}$ shows the lesion volume in the 4 experimental groups. $\mathrm{SE}=$ standard error. ${ }^{*} \mathrm{p}<0.05$ vs MCAo. 


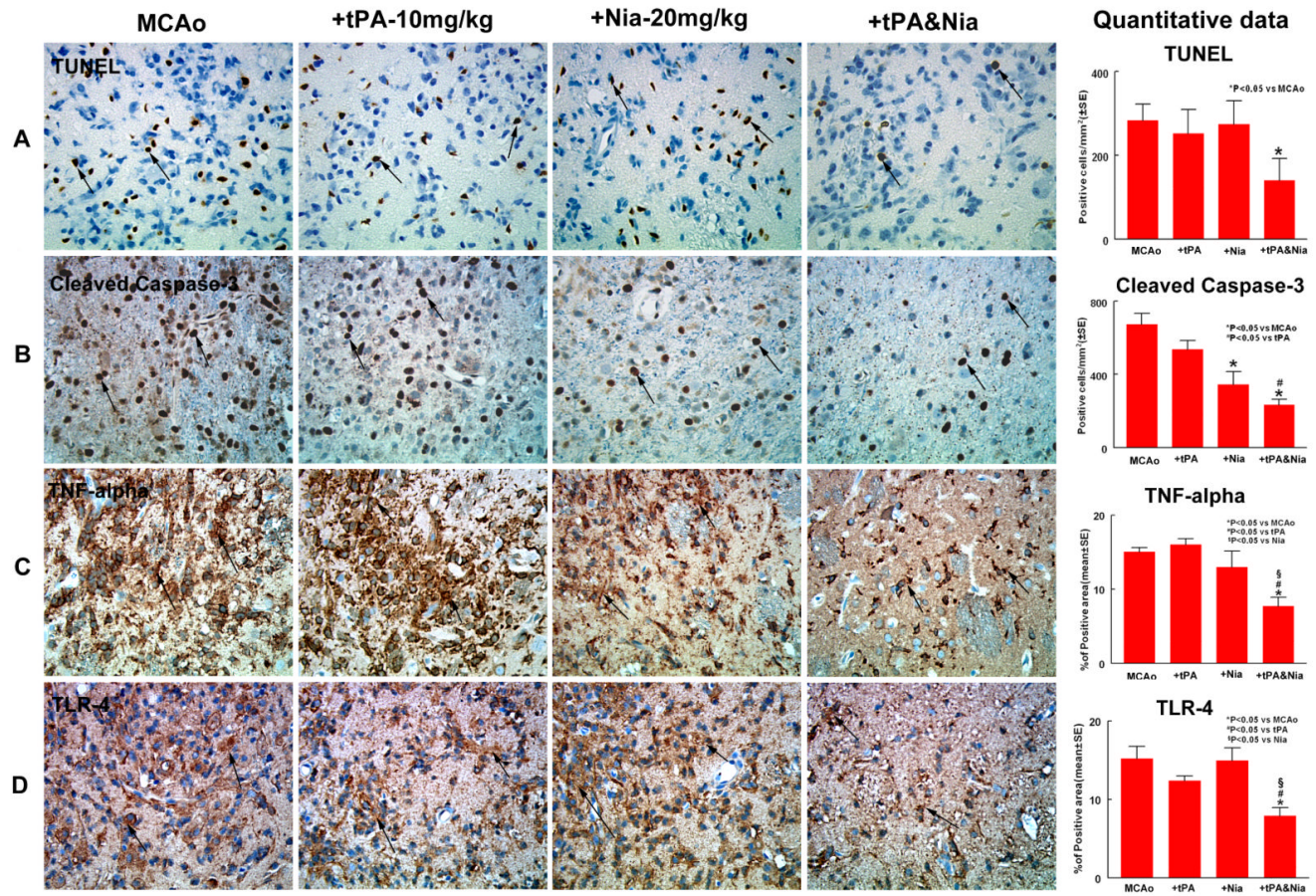

Figure 2. Combination treatment with Niaspan and tPA reduces apoptosis and attenuates TNFalpha and TLR-4 expression

Panels A-B show that combination treatment significantly decreased the number of TUNELpositive (A) and cleaved caspase-3 (B) expression in the ischemic brain compared to saline treated rats $(\mathrm{p}<0.05)$. Panels $\mathbf{C}-\mathbf{D}$ show that combination treatment significantly reduced the expression of TNF-alpha (C), and TLR-4 (D) in the ischemic brain compared with tPA or Niaspan monotherapy as well as with the saline control group $(\mathrm{p}<0.05)$. $\mathrm{SE}=$ standard error. 